\title{
Uniform Flow Past a Closed Body at Low Reynolds Number Employing a Novel Matching in a Boundary Element Formulation
}

\author{
Bwebum Cleofas Dang ${ }^{1,2,{ }^{*}}$, Edmund Chadwick ${ }^{1}$ \\ ${ }^{1}$ School of Computing, Science and Engineering \\ University of Salford \\ Greater Manchester \\ M5 4WT, United Kingdom. \\ ${ }^{2}$ Department of Mathematics \\ Faculty of Natural Sciences \\ University of Jos \\ PMB 2084, Jos Plateau State, Nigeria. \\ * Corresponding author: b.c.dang@edu.salford.ac.uk
}

\begin{abstract}
Consider a two dimensional steady low Reynolds number flow past a circular cylinder. A boundary integral representation that matches an outer Oseen flow and inner Stokes flow is given, and the matching error is shown to be smallest when the outer domain is as close as possible to the body. Also, it is shown that as the Greens function is approached, the oseenlet becomes the stokeslet to leading order and has the same order of magnitude error as the matching error. This means a novel boundary integral representation in terms of oseenlets is possible. To test this, a corresponding boundary element code is developed which uses point collocation weighting functions, linear shape functions, two-point Gaussian quadrature with analytic removal of the Greens function singularity for the integrations. The method is compared against various methods for the benchmark problem of flow past a circular cylinder. In particular, the drag coefficient is used for the comparison. The advantage of this method over existing ones is demonstrated and discussed particularly in the Reynolds number range $R e=1 \sim 4$.
\end{abstract}

Keywords: Low Reynolds number, Boundary Element Method (BEM), Viscous fluid dynamics, Matched asymptotic expansion. 


\section{Introduction}

In biological fluid dynamics, the modelling of the motion of macroscopic and microscopic organisms represented by a generic closed swimming body is important, such as flagellated propelled organisms like spermatozoa [14]. In particular, the far-field effect at a centimetre scale is often required. As a first step in this paper, a new boundary element method is developed that also incorporates the far-field matching for low Reynolds number two-dimensional steady flow [1.

The Boundary Element Method (BEM) can be traced back to the 1960's [2], its numerical implementation was made robust with the advent of computers that aid solving sets of integral equations. Partial differential equations can be solved numerically by many different methods such as the Finite Difference Method (FDM) and the Finite Element Method (FEM) which are domain methods. However, in certain circumstances such as this one, if a boundary integral formulation is available, then a formulation based on this such as the Boundary Integral Method (BIM) [4] has advantages. For example, the formulation is expressed on the boundary and so has one dimension less than the domain methods FDM and FEM, making it faster and more accurate. With the development of quadratures and stable discretization, the evaluation of integrals becomes more accurate and efficient [4].

Studies of slow motion of viscous fluid flow past a body in an unbounded domain dates back to the work of Stokes in 1851 [15]. Because of the difficulty in satisfying boundary conditions both at the cylinder surface and the farfield, Stokes draws a conclusion that such a solution does not exist and this hypothesis was later termed Stokes' paradox. Several analytical studies began to emanate, seeking solution to the Stokes' paradox and this include the approximation given by Oseen [11 solved approximately by Lamb 8, [9], and Imai [6]. However, Oseen's approximation assumes linearisation to the free stream velocity which breaks down on the body boundary. To overcome this, the method of matched asymptotic expansions was presented by Proudman and Pearson [13] and Kaplun [7] and it combines linearisation to Stokes flow in the near-field matched to linearisation to Oseen flow in the far-field region. Experimental studies [17] with different qualitative and quantitative results have also been presented, in particular for the benchmark problem of steady flow past a circular cylinder.

Further to different numerical methods used, Yano and Kieda [19] applied a discrete singularity method to solve a two-dimensional flow by distributing 
oseenlets, sources, sinks and vortices in the interior of an obstacle with a least square criterion to satisfy the boundary condition. Their result was benchmarked against the analytic results of Lamb [9], Kaplun [7] and the experiment of Tritton [17] for the drag coefficient. It was revealed that when the Reynolds number is below one $(R e<1)$, there is good agreement, but when the Reynolds number is in the range 1 to 4 the analytical results do not align very close with experiment except the numerical studies presented by Yano and Kieda [19. The analytical results work well for body surfaces with simple geometries, but as soon as the geometry becomes complicated, numerical approaches provide better basis for analysis. To apply to more complicated geometries, Lee and Leal [10] considered a matched asymptotic expansion method that used Green's integral representations of the velocity. Chadwick [1] takes this approach and matched Stokes and Oseen flow within a boundary integral formulation. It was found that the error is least if the matching boundary is on the body itself. Here, it is noted that this approach does not break down on the body boundary because in the formulation the oseenlet approximates to the stokeslet.

In this paper, the above mentioned approach in Chadwick [1] is tested by developing a BEM using point collocation weighting functions, linear shape functions, and two-point Gaussian quadrature with analytic removal of the Greens function singularity for the integrations. The Green's integral representation of oseenlets are distributed over the boundary surface. The BEM in this study compares favourably with Tritton experiment [17], analytical results of Lamb [9], Kaplun [7], Tomotika [16], and the numerical results of Yano and Kieda [19] for the drag coefficient. Hence, our method is simple yet robust in solving steady two-dimensional flow past a circular cylinder in an unbounded domain.

\section{Formulation of Governing Equations}

The motion of any continuous fluid is governed by the Navier-Stokes equation, and for a creeping flow, a linearisation of the Navier-Stokes equation yields Stokes and Oseen equation which govern a viscous fluid. Hence, away from a body surface the Oseen equation governs the flow in an outer region (see figure 1a) given by

$$
\rho U \frac{\partial u_{i}}{\partial x_{1}}=-\frac{\partial p}{\partial x_{i}}+\mu \frac{\partial^{2} u_{i}}{\partial x_{j} \partial x_{j}}+f_{i},
$$




$$
\frac{\partial u_{i}}{\partial x_{i}}=0,
$$

where Eq. (2) is the continuity equation, $\rho$ is the density of the fluid, $u_{i}$ is the velocity, $p$ is the pressure, $\mu$ is the viscosity, $U$ is the uniform stream velocity, and $f_{i}$ is the applied force. Similarly, near the body Stokes equation governs the flow in an inner region (see figure $1 \mathrm{~b}$ ) given by

$$
0=-\frac{\partial p}{\partial x_{i}}+\mu \frac{\partial^{2} u_{i}}{\partial x_{j} \partial x_{j}}+f_{i}
$$

(1)

$$
\frac{\partial u_{i}}{\partial x_{i}}=0
$$

The viscous forces in Eq. (3) are dominant over the inertial forces, and by dimensionless analysis, the dimensionless Reynolds number tends to zero near the body with length dimension $l$ and $R e=\frac{\rho U l}{\mu} \rightarrow 0$. To apply the Green's integral, it is supposed that an external force is exerted by the body on the fluid such that the applied force is $f_{i}$.

The work of Chadwick [1] considers a matched near-field region using Stokes flow and the far-field using Oseen flow. The common boundary where the matching takes effect, has $L$ as the length dimension of the matched region and it is seen that $R e \frac{L}{l}$ is the error. So the error is reduced by choosing $L=l$ and Oseen flow assumed everywhere in the flow field, as shown in section 4 .

\section{Green's Function for Oseen and Stokes Equation}

The oseenlet is the Green's function of the Oseen equation. In the limit as the Reynolds number tends to zero, the oseenlet approximates to the stokeslet which is the Green's function of the Stokes equation. The drag and lift oseenlet are

$$
u_{i}^{(1)}=\frac{1}{2 \pi \rho U}\left(\frac{\partial}{\partial x_{i}}\left(\ln r+e^{k x_{1}} K_{0}(k r)\right)-2 k e^{k x_{1}} K_{0}(k r) \delta_{i 1}\right),
$$

$$
p^{(1)}=-\frac{1}{2 \pi} \frac{\partial}{\partial x_{1}}(\ln r)
$$


94

and

$$
u_{i}^{(2)}=\frac{1}{2 \pi \rho U} \varepsilon_{i j 3} \frac{\partial}{\partial x_{j}}\left(\ln r+e^{k x_{1}} K_{0}(k r)\right),
$$

95

$$
p^{(2)}=-\frac{1}{2 \pi} \frac{\partial}{\partial x_{2}}(\ln r),
$$

96

103

108

109 where $C_{i}^{(m)}=\frac{\delta_{i 2} \delta_{m 2}}{4 \pi \mu}$

and stokeslet is given by

$$
u_{i}^{(1)}=\frac{1}{4 \pi \mu}\left(\delta_{i 1} \ln r-\frac{x_{1} x_{i}}{r^{2}}\right)(1+\mathcal{O}(k r)),
$$

$$
p^{(1)}=-\frac{1}{2 \pi} \frac{x_{1}}{r^{2}}
$$

$$
u_{i}^{(2)}=\frac{1}{4 \pi \mu}\left(\delta_{i 2} \ln r-\frac{x_{2} x_{i}}{r^{2}}\right)(1+\mathcal{O}(k r))+C_{i},
$$

$$
p^{(2)}=-\frac{1}{2 \pi} \frac{x_{2}}{r^{2}},
$$

where $C_{i}=\frac{\delta_{i 2}}{4 \pi \mu}$. Thus, up to order $k r$ and a constant, the two-dimensional

$$
u_{i}^{(m)}=\frac{1}{4 \pi \mu}\left(\delta_{i m} \ln r-\frac{x_{m} x_{i}}{r^{2}}\right)(1+\mathcal{O}(k r))+C_{i}^{(m)},
$$

$$
p^{(m)}=-\frac{1}{2 \pi} \frac{x_{m}}{r^{2}}
$$




\section{Green's Integral Formulation}

\subsection{Outer Region}

Consider the space $\Sigma$ enclosed by the boundary around and approaching the point $x_{i}$, the body boundary $l_{B}$, and the boundary on the far-field tending to an infinite distance away $l_{\infty}$ (see figure 1). The Green's integral formulation for the Oseen flow [12] can be found by considering the integral,

$$
\begin{aligned}
& \int_{\Sigma}\left(-\rho U \frac{\partial u_{i}^{(m)}(z)}{\partial y_{1}}-\frac{\partial p^{(m)}(z)}{\partial y_{i}}-\mu \frac{\partial^{2} u_{i}^{(m)}(z)}{\partial y_{j} \partial y_{j}}+f_{i}^{(m)}(z)\right) u_{i}(y) d \Sigma \\
& +\int_{\Sigma}\left(-\rho U \frac{\partial u_{i}(y)}{\partial y_{1}}-\frac{\partial p(y)}{\partial y_{i}}+\mu \frac{\partial^{2} u_{i}(y)}{\partial y_{j} \partial y_{j}}-f_{i}(y)\right) u_{i}^{(m)}(z) d \Sigma=0
\end{aligned}
$$

where $y_{i}$ is a vector position of the exterior domain integrated space $\Sigma$ and in this case an area integral with $z_{i}=x_{i}-y_{i}$, so the differential equation for the Green's functions satisfies the conjugate Oseen equation since $\frac{\partial}{\partial y_{j}}=-\frac{\partial}{\partial x_{j}}$ and $f_{i}^{(m)}(z)=\delta(z) \delta_{i m}$ where $\delta(z)$ is the Dirac delta function. 


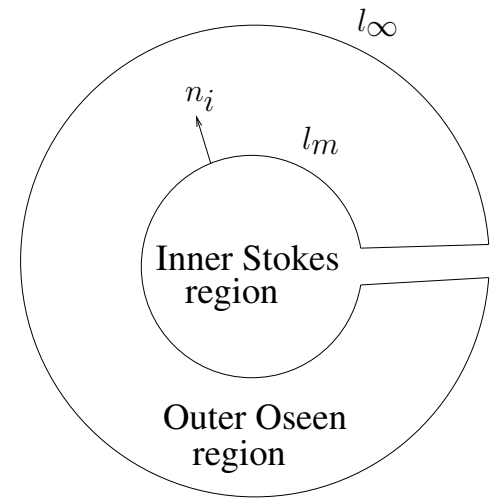

(a) Green's integral representation for outer Oseen flow

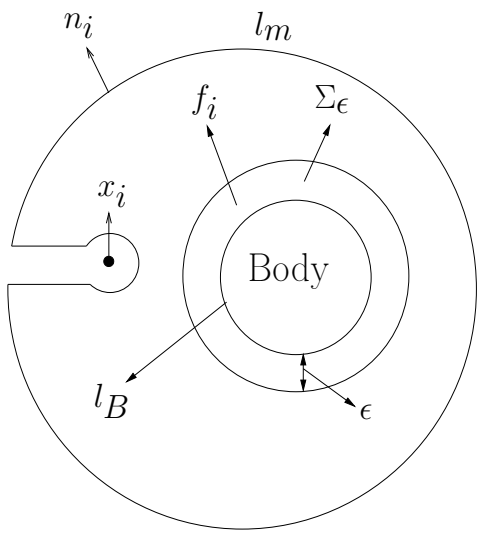

(c) Spatial distribution of point sources

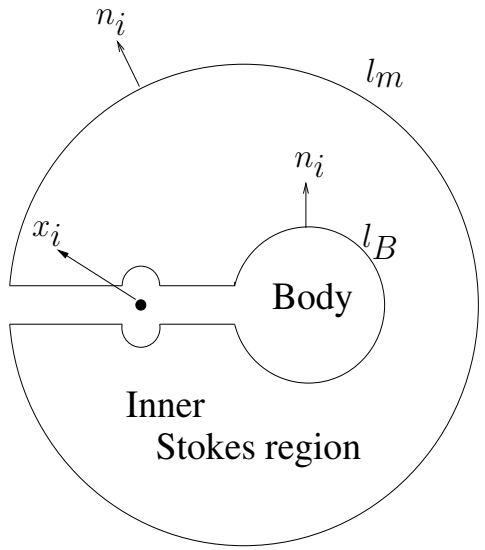

(b) Green's integral representation of inner Stokes flow

Figure 1: Green's integral representation of a body in a near-field and far-field region

In the outer region, there is no body force so $f_{i}=0$ and the point $x_{i}$ is in the inner region, so there is no contribution $f_{i}^{(m)}(z)$ around the point $x_{i}$. 
Rearranging Eq. (15) then gives

$$
\begin{aligned}
0 & =\int_{\Sigma}-\rho U \frac{\partial}{\partial y_{1}}\left(u_{i}^{(m)}(z) u_{i}(y)\right) d \Sigma \\
& -\int_{\Sigma} \frac{\partial}{\partial y_{i}}\left(p^{(m)}(z) u_{i}(y)+p(y) u^{(m)}(z)\right) d \Sigma \\
& +\int_{\Sigma}-\left(\mu \frac{\partial}{\partial y_{j}}\left(\frac{\partial u_{i}^{(m)}(z)}{\partial y_{j}} u_{i}(y)\right)-\mu \frac{\partial}{\partial y_{j}}\left(\frac{\partial u_{i}(y)}{\partial y_{j}} u_{i}^{(m)}(z)\right)\right) d \Sigma .
\end{aligned}
$$

From the continuity equation (Eq. 2), it can be seen that $\mu \frac{\partial u_{i}^{(m)}}{\partial y_{j}} \frac{\partial u_{i}}{\partial y_{j}}$ cancel out in Eq. (16) by applying the divergence theorem. This then gives the Oseen's integral representation as

$$
\begin{aligned}
0 & =\int_{l_{m}}\left(\rho U u_{i}^{(m)}(z) u_{i}(y) n_{1}+\left(p^{(m)}(z) u_{i}(y)+p(y) u_{i}^{(m)}(z)\right) n_{i}\right) d l \\
& +\int_{l_{m}} \mu\left(\frac{\partial u_{i}^{(m)}(z)}{\partial y_{j}} u_{i}(y)-\frac{\partial u_{i}(y)}{\partial y_{j}} u_{i}^{(m)}(z)\right) n_{j} d l
\end{aligned}
$$

where $l_{m}$ is the matching boundary. From Fishwick and Chadwick [3] the far field integral bounding the exterior domain $\Sigma$ in the Oseen representation is zero, where the boundary of the domain in two-dimension is a closed curve.

\subsection{Inner Region}

The same approach used in the preceding section can be applied to give the Green's integral representation for the inner Stokes flow over a different domain integral (see figure 1b). Again there is no body force, so $f_{i}=0$, but there is a contribution around the point $x_{i}$. Rearranging and simplifying Eq. (15) to get

$$
\begin{aligned}
-\int_{\Sigma} f_{i}^{(m)}(z) u_{i}^{s}(y) d \Sigma & =-\int_{\Sigma} \delta(z) \delta_{i m} u_{i}^{s}(y) d \Sigma \\
& =-u_{m}^{s}(x),
\end{aligned}
$$

where $u_{i}^{s}(x), p^{s}(x), u_{i}^{(m) s}(x)$ and $p^{(m) s}(x)$ are the inner Stokes velocity and pressure, and inner Stokeslet velocity and pressure respectively. This then 
gives

$$
\begin{aligned}
-u_{m}^{s}(x) & =\int_{\Sigma}-\rho U \frac{\partial}{\partial y_{1}}\left(u_{i}^{(m) s}(z) u_{i}^{s}(y) \frac{\partial}{\partial y_{i}}\left(p^{(m) s}(z) u_{i}^{s}(y)+p^{s}(y) u^{(m) s}(z)\right)\right) d \Sigma \\
& +\int_{\Sigma}-\mu \frac{\partial}{\partial y_{j}}\left(\left(\frac{\partial u_{i}^{(m) s}(z)}{\partial y_{j}} u_{i}^{s}(y)\right)+\left(\frac{\partial u_{i}^{s}(y)}{\partial y_{j}} u_{i}^{(m) s}(z)\right)\right) d \Sigma,
\end{aligned}
$$

131

$$
\begin{aligned}
u_{m}^{s}(x) & =-\int_{l_{B}}\left(p^{(m) s}(z) u_{i}^{s}(y)+p^{s}(y) u_{i}^{(m) s}(z)\right) n_{i} d l \\
& -\int_{l_{B}} \mu\left(\frac{\partial u_{i}^{(m) s}(z)}{\partial y_{j}} u_{i}^{s}(y)-\frac{\partial u_{i}^{s}(y)}{\partial y_{j}} u_{i}^{(m) s}(z)\right) n_{j} d l \\
& +\int_{l_{m}}\left(p^{(m) s}(z) u_{i}^{s}(y)+p^{s}(y) u_{i}^{(m) s}(z)\right) n_{i} d l \\
& +\int_{l_{m}} \mu\left(\frac{\partial u_{i}^{(m) s}(z)}{\partial y_{j}} u_{i}^{s}(y)-\frac{\partial u_{i}^{s}(y)}{\partial y_{j}} u_{i}^{(m) s}(z)\right) n_{j} d l .
\end{aligned}
$$

\subsection{Matching Inner and Outer region}

Here the inner and outer region are matched using Eq. (18) and Eq. (17), an error introduced as a result of the matching is giving next. In twodimensions, the constant term $C_{i}^{(m)}$ give the leading order approximation to the velocity oseenlet $\left(1+\mathcal{O}\left(\frac{1}{\ln k r}\right)\right)=\left(1+\mathcal{O}\left(\frac{1}{\ln R e \frac{L}{l}}\right)\right)$ on the matching boundary where $r=\mathcal{O}(L)$. Hence, the matching integral in Eq. (18) is

$$
\begin{aligned}
& \int_{l_{m}}\left(p^{(m) s}(z) u_{i}^{s}(y)+p^{s}(y) u_{i}^{(m) s}(z)\right) n_{i} d l+\int_{l_{m}} \mu\left(\frac{\partial u_{i}^{(m) s}(z)}{\partial y_{j}} u_{i}^{s}(y)-\frac{\partial u_{i}^{s}(y)}{\partial y_{j}} u_{i}^{(m) s}(z)\right) n_{j} d l \\
& \quad \times\left(1+\mathcal{O}\left(\frac{1}{\ln R e \frac{L}{l}}\right)\right)=-\int_{l_{m}}\left(\rho U u_{i}^{(m)}(z) u_{i}(y) n_{1}+\left(p^{(m)}(z) u_{i}(y)+p(y) u_{i}^{(m)}(z)\right) n_{i}\right) d l \\
& \quad+\int_{l_{m}} \mu\left(\frac{\partial u_{i}^{(m)}(z)}{\partial y_{j}} u_{i}(y)-\frac{\partial u_{i}(y)}{\partial y_{j}} u_{i}^{(m)}(z)\right) n_{j} d l=0 .
\end{aligned}
$$

So, to make the error as small as possible, we let $L=l$ and consider Oseen flow everywhere in the flow field. 


\section{Green's Integral for the Boundary Element Method}

Now consider the space $\Sigma$ enclosed by the boundary around the body boundary $l_{B}$ and the boundary on the far-field: an infinite distance away $l_{\infty}$. The body is represented by a distribution of forces $f_{i}$ in the region $\Sigma_{\epsilon}$ which is a distance $\epsilon$ away from the body boundary $l_{B}$ (see figure 1) and Eq. (17) then becomes (up to the error in the matching Eq. (19p)

$$
\begin{aligned}
\int_{\Sigma}\left(-f_{i}^{(m)}(z) u_{i}(y)\right. & \left.+f_{i}(y) u_{i}^{(m)}(z)\right) d \Sigma=\int_{\Sigma}-\rho U \frac{\partial}{\partial y_{1}}\left(u_{i}^{(m)}(z) u_{i}(y)\right) d \Sigma \\
& -\int_{\Sigma} \frac{\partial}{\partial y_{i}}\left(p^{(m)}(z) u_{i}(y)+p(y) u^{(m)}(z)\right) d \Sigma \\
& -\int_{\Sigma}\left(\mu \frac{\partial}{\partial y_{j}}\left(\frac{\partial u^{(m)}(z)}{\partial y_{j}} u_{i}(y)\right)+\mu \frac{\partial}{\partial y_{j}}\left(\frac{\partial u_{i}(y)}{\partial y_{j}} u_{i}^{(m)}(z)\right)\right) d \Sigma \\
& =\int_{l_{\infty}}\left(\rho U u_{i}^{(m)}(z) u_{i}(y) n_{1}+\left(p^{(m)}(z) u_{i}(y)+p(y) u_{i}^{(m)}(z)\right) n_{i}\right) d l \\
& -\int_{l_{\infty}} \mu\left(\frac{\partial u_{i}^{(m)}(z)}{\partial y_{j}} u_{i}(y)-\frac{\partial u_{i}(y)}{\partial y_{j}} u_{i}^{(m)}(z)\right) n_{j} d l=0
\end{aligned}
$$

We let

$$
\int_{\Sigma_{\epsilon}} f_{i}(y) u_{i}^{(m)}(z) d \Sigma=\int_{l_{B}} F_{i}(y) u_{i}^{(m)}(z) d l
$$

on the body boundary so that as $\epsilon \rightarrow 0$, it gives the force on the body as

$$
F_{i}(y)=\lim _{\epsilon \rightarrow 0} \int_{0}^{\epsilon} f_{i}(y) d \epsilon .
$$

Therefore,

$$
\begin{aligned}
u_{m} & =\int_{\Sigma}\left(-f_{i}^{(m)}(z) u_{i}(y)+f_{i}(y) u_{i}^{(m)}(z)\right) d \Sigma \\
& =\int_{\Sigma_{\epsilon}} f_{i}(y) u_{i}^{(m)}(z) d \Sigma
\end{aligned}
$$

Hence,

$$
u_{m}(x)=\int_{l_{B}} F_{i}(y) u_{m}^{(i)} d l
$$

150 because by symmetry, $u_{i}^{(m)}=u_{m}^{(i)}$ from Eq. (5) and Eq. (7).

To proceed with the numerical method, Eq. (24) is discretised in the BEM given next. 


\section{Numerical Method}

In the preceding section, the oseenlet is derived and given in Eq. (24) for a two-dimensional flow satisfying the Oseen equation for the far-field region and it was also shown above that in the matched region the oseenlet becomes the stokeslet. We shall compute the drag experienced by a circular cylinder in a steady flow in an unbounded domain. To do this, Eq. (24) is discretised using the BEM with a point collocation weighting function as seen in figure $2 \mathrm{a}$, where $x_{\alpha i}$ is the position $x_{i}$ of node $\alpha$, the two nodal points are given by $x_{\alpha i}$ and $x_{\alpha+1 i}$, while the midpoint between them is the collocation point. The collocation point is chosen not to lie on the nodes so that the Green's function singularity in the integral is more easily removed, because the singularity lies wholly within the element integration rather than divided across two elements. For ease of numerical formulation, the boundary is approximated by a linear rather than a curved variation, but as the number of nodes are increased the collocation points will move closer to the boundary and so this is not expected to be a problem.

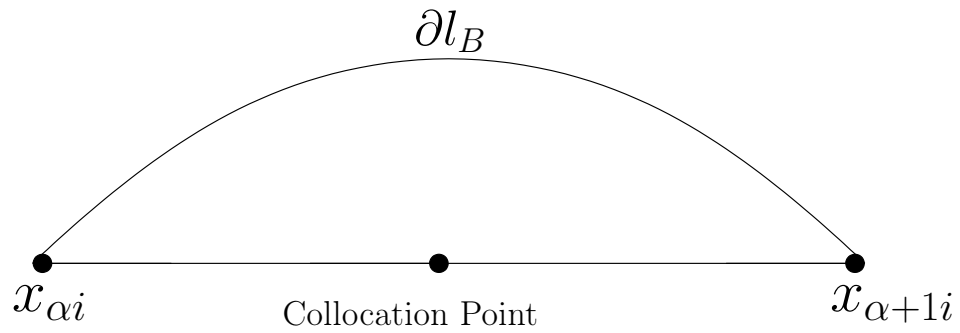

(a) Diagram showing collocation point

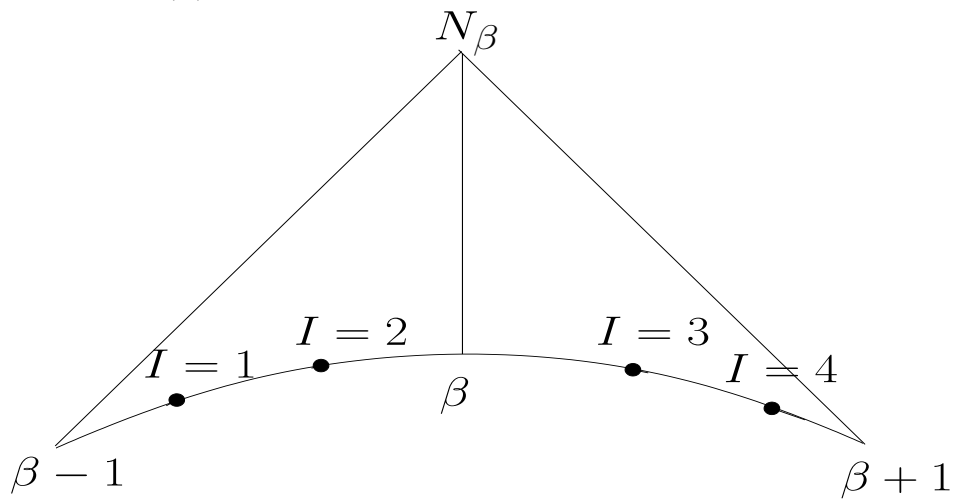

(b) Diagram showing Gaussian points

Figure 2: Figure showing the nodal points and Gaussian points used for collocation 
In figure 2b, a two-point Gaussian quadrature is shown with Gaussian points $I=1,2$ for the integral from node $\beta-1$ to $\beta$, and $I=3,4$ for the integral from node $\beta$ to $\beta+1, N_{\beta}$ is the linear shape function at node $\beta$ and $g p w_{I}$ is the Gaussian point weight at point $I$. Hence Eq. (24) now becomes

$$
\begin{aligned}
u_{i}(x) & =\int_{l_{B}} N_{\beta} f_{\beta j} u_{i}^{(j)} d l \\
& =f_{\beta j} N_{\beta j} u_{i j I} g p w_{I},
\end{aligned} .
$$

where there are implied summations over $1 \leq \beta \leq n$ (for $n$ nodes), over $1 \leq I \leq 4$ (for Gaussian points associated with node $\beta$ (see figure 2), and over $1 \leq j \leq 2$ (for spatial dimension).

Also, $u_{i j I}$ is the value of the oseenlet Green's function $u_{i}^{(j)}$ positioned at the Gaussian point $I$ of node $\beta$, and determined at the node $\alpha$. Hence, this collocation point method transforms the integral equation into a linear system of algebraic equations with a no slip boundary condition yielding

$$
\text { Af }=\mathbf{Y}
$$

where $\mathbf{A}$ is a $2 n \times 2 n$ matrix, $\mathbf{f}$ is the force coefficient and $\mathbf{Y}$ is an $n$ dimensional vector given by applying the boundary condition. Singularities from the Green's function which formed part of the matrix $\mathbf{A}$ are removed analytically. The full numerical formulation, including how the singularity is removed, is put in the appendix.

\subsection{Flow Past a Circular Cylinder}

As a first step of testing the BEM developed here, we begin by plotting streamlines for a flow past a circular cylinder. Although figure 3 and figure 4 do not give any quantifiable information, but they do give visualization for the streamlines in Reynolds number range 0.01 to 4 as expected from experiment, giving confidence in the formulation. It was noticed that for $R e=0.01$, the streamlines align (see figure 3). Whereas when the Reynolds number is increase to about 4, eddies began to form near the cylinder (see figure 4) which is expected from experiment [18]. This is benchmark against analytical results for low Reynolds number below 0.1 , given accuracy of $1 \%$. 


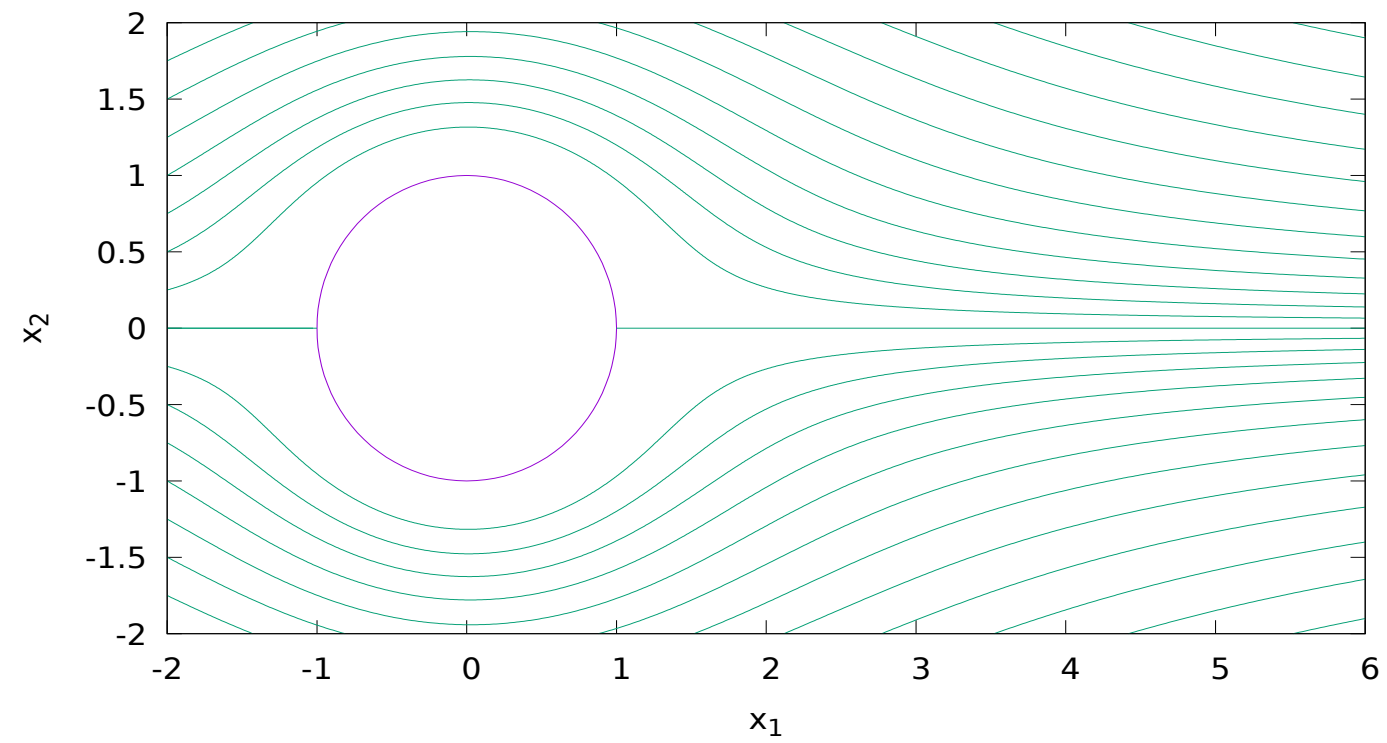

Figure 3: Streamlines of steady flow past a circular cylinder at $R e=0.01$ in an unbounded domain.

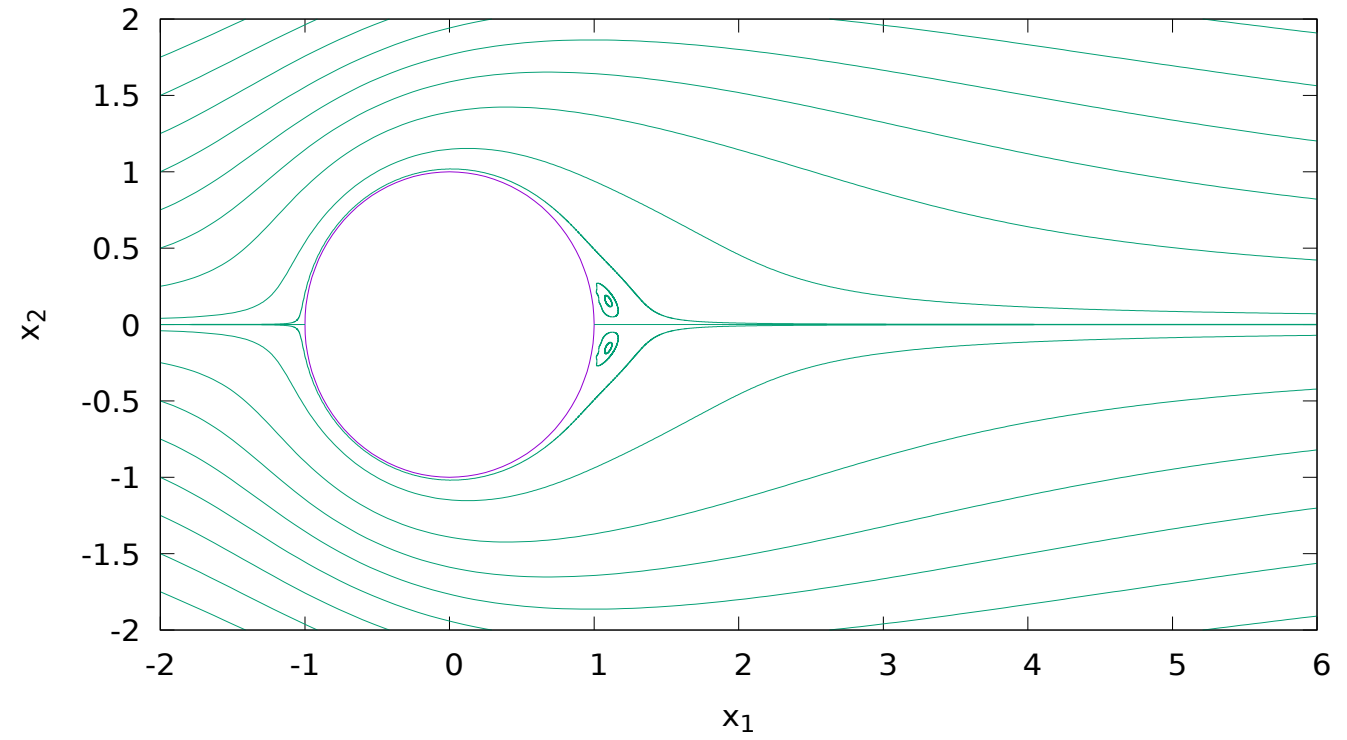

Figure 4: Streamlines of steady flow past a circular cylinder at $R e=4$ in an unbounded domain which formed eddies 
To validate against existing results, the drag coefficient $C_{D}$ from the BEM presented in this study is compared against results of Lamb [9] Eq. (27), Tomotika [16] Eq. (28), Kaplun [7] Eq. (29), experimental results of Tritton [17], and numerical results of Yano and Kieda [19] all for a Reynolds number Re ranging between 0 and 4 (see figure 5). The approximation of the drag coefficients for the various listed results are

$$
\text { Lamb: } \quad C_{D}=\frac{4 \pi}{R e T_{1}}
$$

$$
\text { Tomotika: } \quad C_{D}=\frac{4 \pi}{R e T_{1}}\left(1-T_{2}\right)
$$

$$
\text { Kaplun: } \quad C_{D}=\frac{4 \pi}{R e T_{1}}\left(1-0.87 T_{1}^{-2}\right)
$$

$$
\text { Lee and Leal: } \quad C_{D}=\frac{-2 \pi}{R e \ln (2 R e)}\left(1+\frac{1}{\ln (2 R e)}\left(\frac{1}{2}-\gamma+\ln 4\right)\right)
$$

where the Reynolds number $R e$ is defined by $R e=\frac{a U}{\nu}$, with $a$ as the cylinder radius and $\nu=\frac{\mu}{\rho}$ is the kinematic viscosity with $\mu$ as the dynamic viscosity of the ambient fluid. The parameter $T_{1}=\left(\frac{1}{2}-\gamma-\log \frac{R e}{4}\right)^{-1}$, $T_{2}=\frac{R e^{2}}{8 T_{1}}\left(T_{1}^{2}-\frac{1}{2} T_{1}+\frac{5}{16}\right)$ with $\gamma=0.577216 \ldots$ as the Euler constant. 


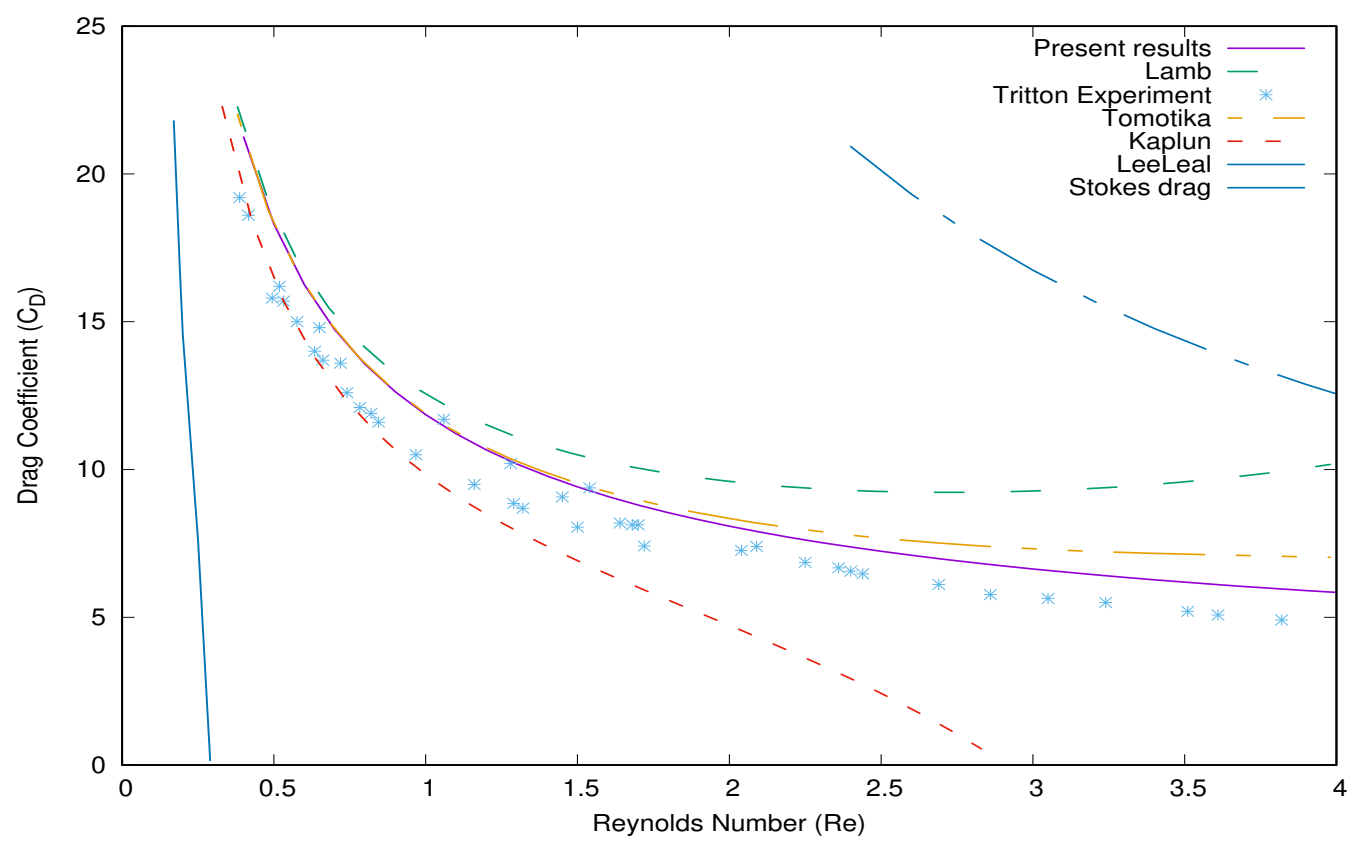

Figure 5: Drag coefficient $C_{D}$ are plotted against the Reynolds number $(0<R e<4)$

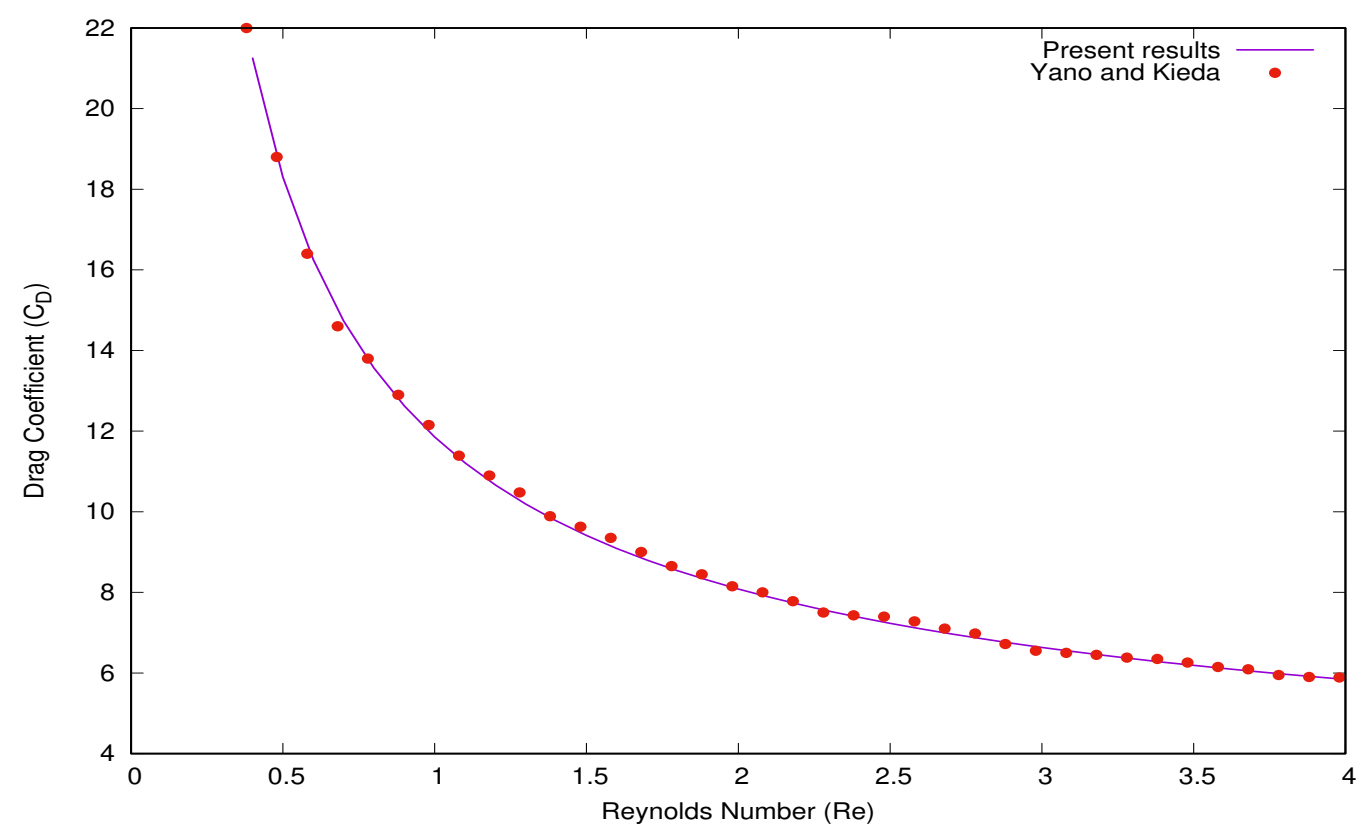

Figure 6: Comparing present result with Yano and Kieda [19] 
In figure 5, the drag coefficient is plotted against the Reynolds number. Lamb's [9] and Kaplun's [7 vary increasingly as the Reynolds number is increased beyond $1(R e>1)$, and the present results together with Yano and Kieda [19] give the closest match to Tritton's experiment [17]. The Stokes drag shows clearly that the velocity diverges when considering a 2D flow past a circular cylinder in an unbounded domain as expected from Stokes paradox. When considering the Reynolds number below $1(R e<1)$, it can be seen that the difference in the results are not significant (see figure 5), they all aligned with experiment at very low Reynolds number. Analytic result of Kaplun and Lagerstrom actually diverge to a negative value as the Reynolds number increase above $2.9(R e>2.9)$.

Furthermore, the present result is compared with the discrete singularity result of Yano and Kieda [19] at similar range of Reynolds number (see figure 6). In their formulation, Yano and Kieda choose a specific points within a body surface and distributed oseenlets, sink, and sources within a body. It is unclear on how to extend the work of Yano and Kieda [19] to a general closed body as their method specifically tailored to the circular cylinder, whereas the method presented here is straightforward to apply for any closed body. 


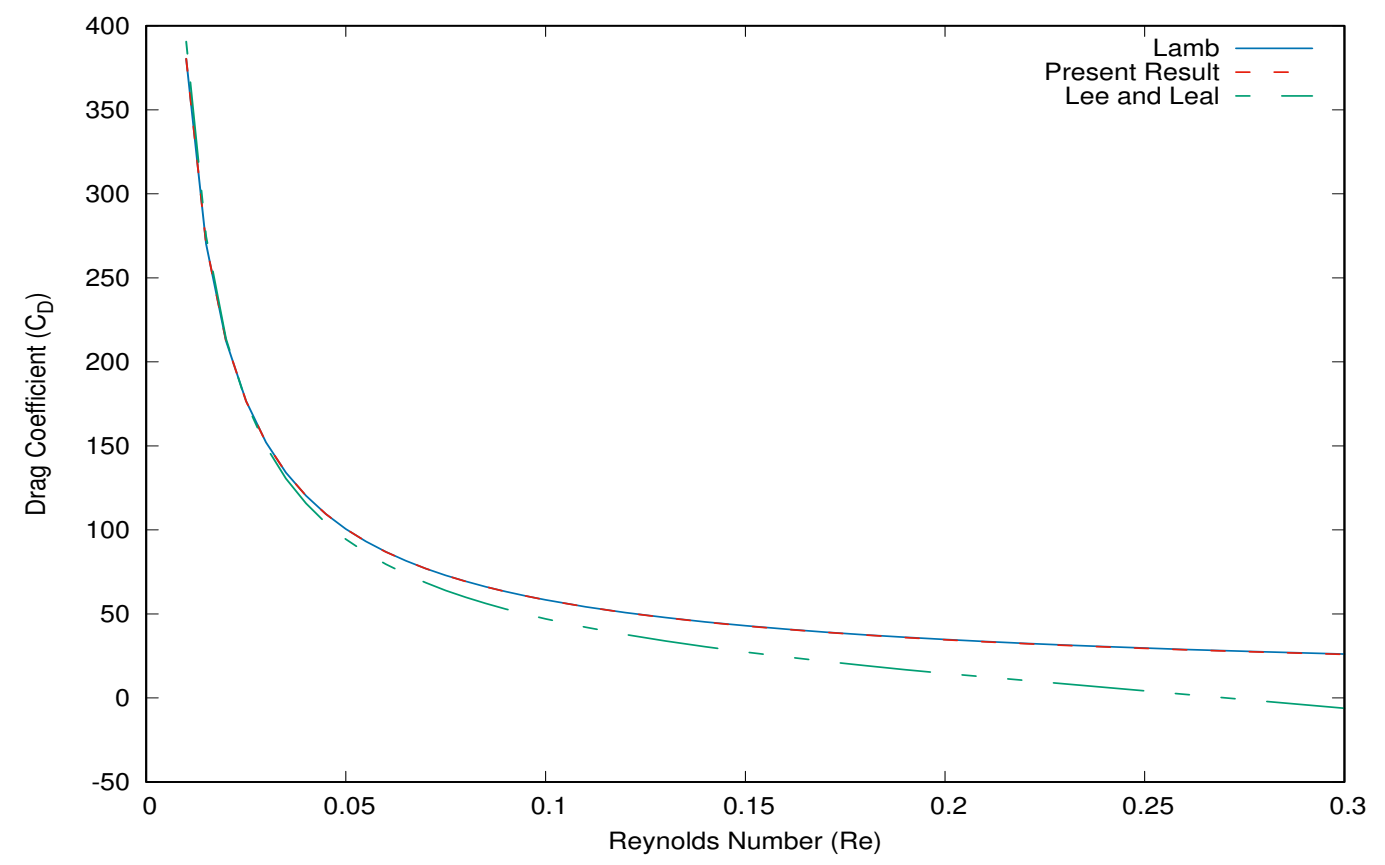

Figure 7: Comparisons of drag coefficient for very low $R e$ in range $0.01 \leq R e \leq 0.3$ for Lamb, Lee and Leal/Proudman and Pearson, and our BEM

Observe that in figure 7, Lamb [8] and present result appear the same in the range $0.01<R e<0.3$, but the result of Lee and Leal [10] begin to diverge as the Reynolds number increases. The result of Lee and Leal diverges to negative values when $R e>0.28$.

\subsection{Flow Past an Elliptical Cylinder}

The BEM developed here is also tested on elliptical cylinder at different angle of inclination ranging from $0^{\circ}$ to $90^{\circ}$. In figures 8 and 9 , we consider the thickness ratio of the elliptic cylinder denoted by $t$, which is the ratio of the minor axis to major axis of the ellipse. The figures are shown for the drag coefficient against angle of attack $\alpha$, varying from $0^{\circ}$ to $90^{\circ}$ for the ellipse. In figure (8), the Reynolds number is set to $R e=0.1$. When $t=1$, it can be seen that the drag coefficient remains constant irrespective of the angle $\alpha$, it is true because that gives a circular cylinder. When $t=0.1$ and $t=0.5$ it can be seen that the drag coefficient reaches optimal when the angle is $90^{\circ}$, this is expected when compared to the results of Yano and Kieda [19]. In figure 9, the Reynolds number is now set to $R e=1$ with the same angle of attack 
242

as in figure (8), it can be seen that the drag coefficient here is lower but it also reaches optimal drag values when the angle is $90^{\circ}$. The drag coefficient here is lower than when the Reynolds number is $R e=0.1$ which is expected.

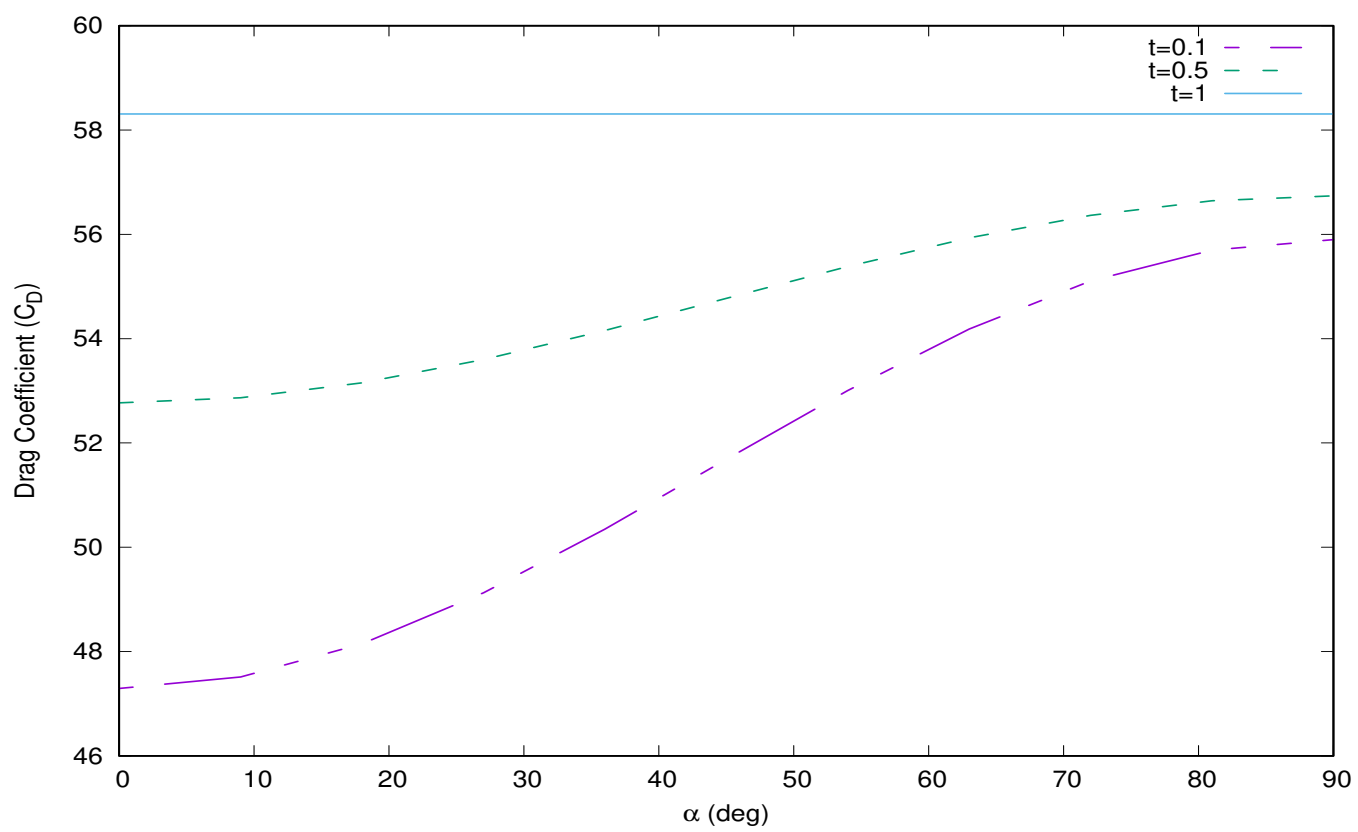

Figure 8: Drag coefficient $C_{D}$ for an inclined elliptical cylinder at Reynolds number $R e=$ 0.1 plotted against angle $\alpha$ for present result 


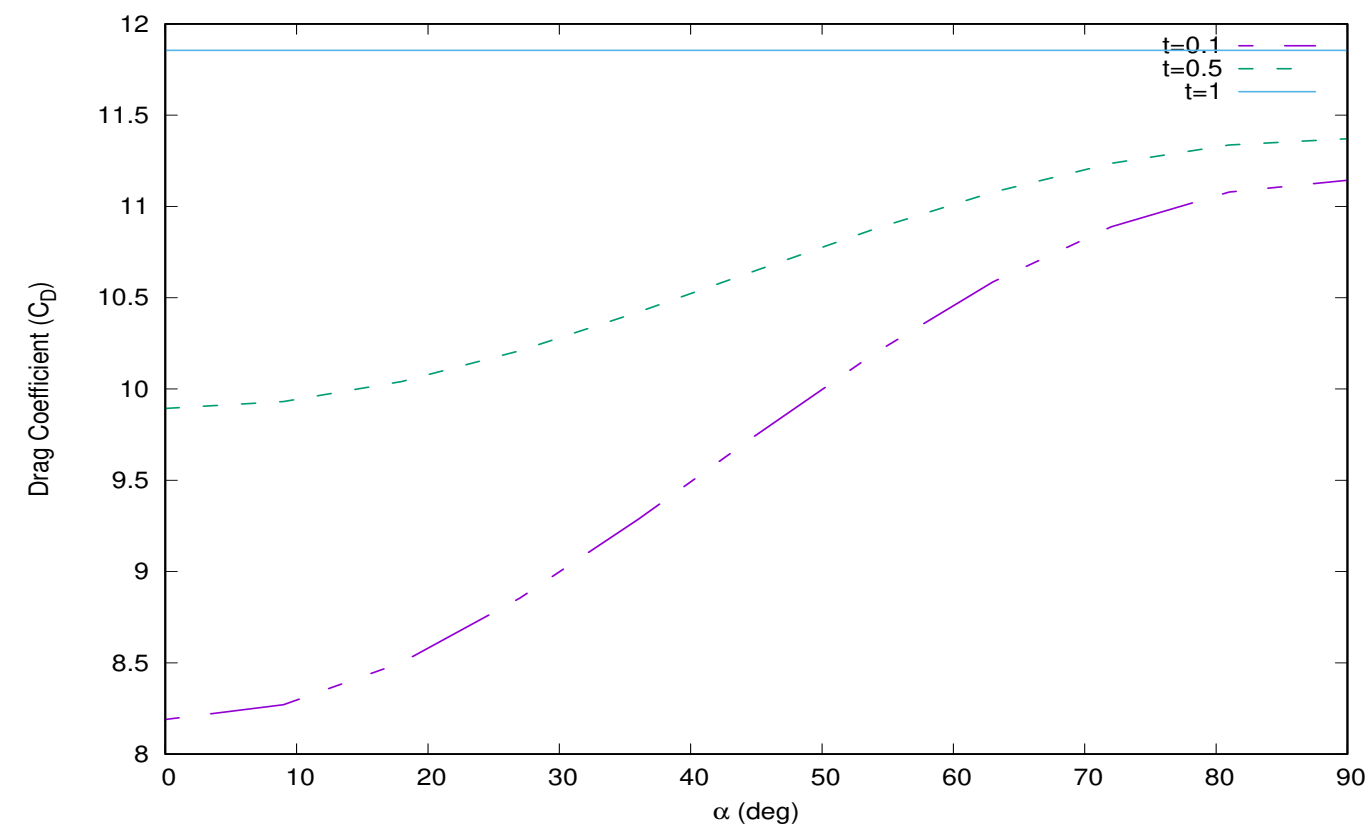

Figure 9: Drag coefficient $C_{D}$ for an inclined elliptical cylinder at Reynolds number $R e=1$ plotted against angle $\alpha$ for present result

Hence matching Stokes and Oseen equation in a boundary element formulation using point collocation weighting functions, linear shape functions, two-point Gaussian quadrature with analytic removal of the Green's function singularity for the integrations give good results compared to other methods discussed.

\section{Conclusion}

A BEM for solving a two-dimensional steady flow past a circular cylinder has been presented. Our results agree against the other benchmark results and are an improvement at the higher Reynolds number range up to 4 . So our representation gives a good description of the flow field even outside the low-Reynolds number region of $R e<1$. In particular, it gives better results than the matched asymptotic method of Kaplun [7]. The present result is also able to deal with complicated geometries. For future work, consider biological fluid dynamics and the modelling of the motion of macroscopic organisms, microscopic organisms, and micro robots. Such an organism can 
be represented by a generic closed swimming body in a quasi-steady problem by using this boundary element method.

\section{Appendix}

\subsection{Numerical Formulation}

The discretisation leading to Eq. (25) is

$$
\begin{aligned}
u_{i} & =\int_{\partial \Sigma_{0}} f_{j} u_{i j} d l^{\prime} \\
& =\int_{\partial \Sigma_{0}} N_{\beta} f_{\beta j} u_{i j} d l^{\prime}
\end{aligned}
$$

where $N_{\beta}\left(\underline{x}^{\prime}\right)$ is the shape function, $u_{i j}\left(\underline{x}-\underline{x}^{\prime}\right)$ is the Green's function evaluated at $\underline{x}^{\prime}, \underline{x}^{\prime}$ is a position on the domain $\partial \Sigma_{0}, d l^{\prime}$ is an element of the length integration variable. $1 \leq i, j \leq m$, where $m$ is the size of the dimensional space and $1 \leq \beta \leq n$ represents the descritisation points.

On the boundary,

$$
\int_{\partial \Sigma_{0}} W_{\alpha} u_{i} d l=\int_{\partial \Sigma_{0}} W_{\alpha} \int_{\partial \Sigma_{0}} N_{\beta} f_{\beta j} u_{i j} d l^{\prime} d l
$$

where $1 \leq \alpha \leq n, W_{\alpha}(\underline{x})$ is the weighting function at node $\alpha$ integrated over $\underline{x}$ position on $\Sigma$ element of length $d l$.

As a result,

$$
u_{\alpha i}=u_{\alpha \beta i j} f_{\beta j}
$$

where

$$
\begin{gathered}
u_{\alpha i}=\int_{\partial \Sigma_{0}} W_{\alpha} u_{i} d l \\
u_{\alpha \beta i j}=\int_{\partial \Sigma_{0}} W_{\alpha} \int_{\partial \Sigma_{0}} N_{\beta} u_{i j} d l^{\prime} d l .
\end{gathered}
$$

We need to renumber 32 so that we can put it into a matrix form in order to solve it in a matrix solver.

Hence, we renumber to $\alpha^{*}=\alpha+(i-1) n$, and $\alpha=\alpha^{*}-(i-1) n$, with $\beta^{*}=\beta+(j-1) n$, and $\beta=\beta^{*}-(j-1) n$, where $1 \leq \alpha^{*}, \beta^{*} \leq m \times n$, $i=1+\left(\frac{\alpha^{*}}{n+1}\right)_{\text {integer division, }}$, and $j=1+\left(\frac{\beta^{*}}{n+1}\right)_{\text {integer division }}$.

In renumbered form, 32 becomes

$$
u_{\alpha^{*}}=u_{\alpha^{*} \beta^{*}} f_{\beta^{*}}
$$


273

and the matrix we require is

$$
f_{\beta^{*}}=u_{\alpha^{*} \beta^{*}}^{-1} u_{\alpha^{*}}
$$

Consider a uniform flow $\delta_{i 1}$ past a two dimensional $(m=2)$ circular cylinder of radius 1, given that the weighting function is the collocation point and the shape function is a linear two-point Gaussian, we want to evaluate $u_{\alpha^{*}}\left[u_{\alpha i}\right]$

$$
\begin{aligned}
u_{\alpha i} & =\int_{\partial \Sigma_{0}} W_{\alpha} u_{i} d l \\
& =\int_{\partial \Sigma_{0}} \delta\left(\underline{x}_{\alpha+\frac{1}{2}}\right) u_{i} d l \\
& =u_{i}\left(\underline{x}_{\alpha+\frac{1}{2}}\right)
\end{aligned}
$$

The last term on the above equation is the mid point shown in figure $2 \mathrm{a}$.

For clarity purposes, $\underline{x}_{\alpha} / x_{\alpha i}$ is a position vector $x_{i}$ of node $\alpha$ and $\underline{x}_{\alpha+\frac{1}{2}} / x_{\alpha+\frac{1}{2} i}$ is the position vector $x_{i}$ of the mid-point between nodes $\alpha$ and $\alpha+1$. $x_{\alpha+\frac{1}{2} i}=$ $\frac{1}{2}\left(x_{\alpha i}+x_{\alpha+1 i}\right)$ is the mid-point with the boundary condition $\left.u_{i}\right|_{\partial \Sigma_{0}}=-\delta_{i 1}$, which means that $u_{\alpha i}=u_{\alpha+\frac{1}{2} i}=-\delta_{i 1}$. We also want to evaluate the Nondegenerative singularity case, first, for $\alpha \neq \beta-1, \beta$ which gives

$$
\begin{aligned}
u_{\alpha \beta i j} & =\int_{\partial \Sigma_{0}} W_{\alpha} \int_{\partial \Sigma_{0}} N_{\beta} u_{i j} d l^{\prime} d l \\
& =\int_{\partial \Sigma_{0}} N_{\beta} u_{i j}\left(\underline{x}_{\alpha+\frac{1}{2}}-\underline{x}\right) d l^{\prime} \\
& =N_{\beta I} g p w_{I} u_{i j}(\underline{y}),
\end{aligned}
$$

where $y_{i}=x_{\alpha+\frac{1}{2} i}-x_{\beta i I}, N_{\beta I}$ is the shape function at Gaussian points $I, N_{\beta I}=$ $\left(\frac{1}{2}-\frac{1}{2 \sqrt{3}}, \frac{1}{2}+\frac{1}{2 \sqrt{3}}, \frac{1}{2}+\frac{1}{2 \sqrt{3}}, \frac{1}{2}-\frac{1}{2 \sqrt{3}}\right), g p w_{I}$ is the Gaussian point weight at point $I$ with $g p w_{I}=\left(\frac{l^{-}}{2}, \frac{l^{-}}{2}, \frac{l^{+}}{2}, \frac{l^{+}}{2}\right)$ where the length between nodes is given as $l^{-}=\left|x_{\beta i}-x_{\beta+1 i}\right|$, and $l^{+}=\left|x_{\beta+1 i}-x_{\beta i}\right|$. The four different Gaussian points are illustrated in figure $2 \mathrm{~b}, x_{\beta i I}$ is the position $x_{i}$ of Gaussian point $I$ 
of node $\beta$ such that

$$
\begin{aligned}
x_{\beta i I}= & \frac{x_{\beta-1 i}+x_{\beta i}}{2}-\frac{x_{\beta i}-x_{\beta-1 i}}{2 \sqrt{3}}, \\
& \frac{x_{\beta-1 i}+x_{\beta i}}{2}+\frac{x_{\beta i}-x_{\beta-1 i}}{2 \sqrt{3}}, \\
& \frac{x_{\beta+1 i}+x_{\beta i}}{2}-\frac{x_{\beta+1 i}-x_{\beta i}}{2 \sqrt{3}}, \\
& \frac{x_{\beta+1 i}+x_{\beta i}}{2}+\frac{x_{\beta+1 i}-x_{\beta i}}{2 \sqrt{3}}
\end{aligned}
$$

${ }_{277} u_{i j}(y)$ is the stokeslet given by

$$
u_{i j}(y)=\frac{R e}{4 \pi}\left(\delta_{i j} \ln r-\frac{y_{i} y_{j}}{r^{2}}\right)
$$

and where $r=+\sqrt{y_{i} y_{j}}$.

We also wish to evaluate the degenerate case with singularities for $i=j$, $\alpha=\beta$. In this case, the singularity needs to be removed

$$
\begin{aligned}
u_{\alpha \beta i j} & =\int_{\partial \Sigma_{0}} N_{\beta} u_{i j} d l^{\prime} \\
& =\int_{l^{-}} N_{\beta} u_{i j} d l^{\prime}+\int_{l^{+}} N_{\beta}\left(u_{i j}-u_{i j}^{s^{*}}\right) d l^{\prime}+\int_{l^{+}} N_{\beta} u_{i j}^{s^{*}} d l^{\prime} \\
& =N_{\beta 1} u_{i j}(\underline{y}) g p w_{1}+N_{\beta 2} u_{i j}(\underline{y}) g p w_{2}+N_{\beta 3}\left(u_{i j}-u_{i j}^{s^{*}}\right) g p w_{3} \\
& +N_{\beta 4}\left(u_{i j}-u_{i j}^{s^{*}}\right)+\int_{l^{+}} N_{\beta} u_{i j}^{s^{*}} d l^{\prime},
\end{aligned}
$$

where $s^{*}$ denotes a singularity, and when the singularity is solved analytically,

$$
u_{i j}=\frac{R e}{4 \pi} \delta_{i j} \ln r .
$$

Thus,

$$
\begin{aligned}
\int_{l^{+}} N_{\beta} u_{i j}^{s^{*}} d l^{\prime} & =\frac{R e}{4 \pi} \int_{l^{+}} N_{\beta} \ln r d l^{\prime} \\
& =\frac{R e}{4 \pi} \delta_{i j}\left(\frac{l^{+}}{2}\left(\ln \left(\frac{l^{+}}{2}\right)-1\right)\right),
\end{aligned}
$$


and when $i=j$ and $\alpha=\beta-1$, then

$$
\begin{aligned}
u_{\alpha \beta i j} & =\int_{\partial \Sigma_{0}} N_{\beta} u_{i j} d l^{\prime} \\
& =\int_{l^{-}} N_{\beta}\left(u_{i j}-u_{i j}^{s^{*}}\right) d l^{\prime}+\int_{l^{-}} N_{\beta} u_{i j}^{s^{*}} d l^{\prime}+\int_{l^{+}} N_{\beta} u_{i j} d l^{\prime} \\
& =N_{\beta 1}\left(u_{i j}-u_{i j}^{s^{*}}\right) g p w_{1}+N_{\beta 2}\left(u_{i j}-u_{i j}^{s^{*}}\right) g p w_{2} \\
& +\int_{l^{-}} N_{\beta} u_{i j}^{s^{*}} d l^{\prime}+N_{\beta 3} u_{i j} g p w_{3}+N_{\beta 4} u_{i j} g p w_{4}
\end{aligned}
$$

so that we have

$$
\begin{aligned}
\int_{l^{-}} N_{\beta} u_{i j}^{s^{*}} d l^{\prime} & =\frac{R e}{4 \pi} \delta_{i j} \int_{l^{-}} N_{\beta} \ln r d l^{\prime} \\
& =\frac{R e}{4 \pi} \delta_{i j}\left(\frac{l^{-}}{2}\left(\ln \left(\frac{l^{-}}{2}\right)-1\right)\right) .
\end{aligned}
$$

To find the solution to (39), we shall find the velocity in the domain, pressure coefficient on the cylinder, as well as the drag coefficient.

In the fluid, the velocity becomes

$$
\begin{aligned}
u_{i}(\underline{x}) & =\int_{\partial \Sigma_{0}} N_{\beta} f_{\beta j} u_{i j} d l^{\prime} \\
& \approx N_{\beta I} f_{\beta j} u_{i j}\left(\underline{x}-\underline{x}_{\beta I}\right) g p w_{I} .
\end{aligned}
$$

By linear superposition,

$$
p(\underline{x}) \approx f_{\beta j} N_{\beta I} p_{j}\left(\underline{x}-\underline{x}_{\beta I}\right) g p w_{I}
$$

${ }_{281}$ where $p_{j}$ is the Stokes pressure given by

$$
p_{j}=\frac{-1}{2 \pi} \frac{y_{j}}{r^{2}}
$$

282

On the cylinder, the pressure at node $\beta$ is

$$
p_{\beta}=-\left.f_{\beta j} n_{j}\right|_{\beta}
$$


where $\left.n_{j}\right|_{\beta}=x_{\beta j}$.

The force coefficient:

$$
\begin{aligned}
C_{i} & =\int_{\partial \Sigma_{0}} f_{i} d l \\
& \approx \int_{\partial \Sigma_{0}} N_{\beta} f_{\beta i} d l \\
& \approx f_{\beta i} N_{\beta i} g p w_{I} \\
& =f_{\beta i}\left(s_{\beta}\left(\frac{l^{+}+l^{-}}{2}\right)\right) \\
& =f_{\beta i} s_{\beta} L
\end{aligned}
$$

where $s_{\beta}=1$ is the summation vector and $l=\frac{l^{+}+l^{-}}{2}$ for $n$ nodes. When $l^{-}=l^{+}=l$, then $l=\frac{2 \pi}{n}$, and

$$
\begin{aligned}
C_{i} & =\frac{2 \pi}{n} f_{\beta i} s_{\beta} \\
& =\frac{2 \pi}{n} \sum_{\beta=1}^{n} f_{\beta i} .
\end{aligned}
$$

Where $i=1$, equation (47) describes the drag coefficient, while for $i=2$, it describes the lift coefficient.

These numerical results must be tested against known analytical solutions. The analytical solutions are

$$
u_{i}=\frac{8 \pi}{R e} u_{i 1}+\frac{2 \pi}{R e} u_{i 1, j j}
$$

and

$$
p=\frac{8 \pi}{R e} p_{1}+\frac{2 \pi}{R e} p_{1, j j},
$$

so the analytical solution is represented by a drag stokeslet of strength $\frac{8 \pi}{R e}$ plus a quadrupole giving drag, such that

$$
C_{D}=\frac{8 \pi}{R e} .
$$

Recall that the stokeslet velocity and pressure are given as

$$
u_{i j}=\frac{R e}{4 \pi}\left(\delta_{i j} \ln r-\frac{y_{i} y_{j}}{r^{2}}\right),
$$




$$
p_{j}=-\frac{1}{2 \pi} \frac{y_{j}}{r^{2}}
$$

and the Stokes equation given by

$$
0=-p_{, i}+\frac{1}{R e} u_{i, j j}
$$

Therefore, the velocity is shown to be a uniform stream, given by

$$
\begin{aligned}
\left.u_{i}\right|_{r=1} & =\left[\frac{8 \pi}{R e} u_{i 1}+2 \pi p_{1, i}\right]_{r=1} \\
& =\left[\frac{8 \pi}{R e}\left(\frac{R e}{4 \pi}\left(\delta_{i j} \ln r-\frac{y_{i} y_{j}}{r^{2}}\right)\right)+2 \pi\left(-\frac{1}{2 \pi} \frac{y_{j}}{r^{2}}\right)_{, i}\right]_{r=1} \\
& =\left[2 \delta_{i 1} \ln r-\frac{2 y_{i} y_{1}}{r^{2}}-\frac{r^{2} \delta_{i 1}-y_{i} 2 r y_{i} / r}{r^{4}}\right]_{r=1} \\
& =\left[2 \delta_{i 1} \ln r-\frac{2 y_{i} y_{1}}{r^{2}}-\frac{\delta_{i 1}}{r^{2}}+\frac{2 y_{i} y_{1}}{r^{4}}\right]_{r=1} \\
& =-\delta_{i 1} .
\end{aligned}
$$

\section{Acknowledgement}

This work is supported by the Nigerian government and the University of Jos, Plateau State, Nigeria.

\section{References}

[1] Chadwick, E.A.: The Far-field Greens Integral in Stokes Flow from the Boundary Integral Formulation. CMES. 96(3), (2013) 177-184.

[2] Cheng, A.H-D. and Cheng, D.T.: Heritage and early history of the boundary element method. Engineering Analysis with Boundary Elements 29(3) (2005), 268-302.

[3] Fishwick, N. and Chadwick, E.: The evaluation of the far-field integral in the Greens function representation for steady Oseen flow. Physics of Fluids 18(11) (2006), 113101.

[4] Hao, W., Hu, B., Li, S., and Song, L.: Convergence of boundary integral method for a free boundary system. Journal of Computational and Applied Mathematics 334 (2018), 128-157. 
[5] Imai, I.: On the asymptotic behaviour of viscous fluid flow at a great distance from a cylindrical body, with special reference to Filons paradox. Proc. Roy. Soc. Lon. A. 208(1095) (1951), 487-516.

[6] Imai, I.: A new method of solving Oseen's equation and it's application to the flow past an elliptic cylinder. Proc. Roy. Soc. Lon. A. 224 (1954), 141-160.

[7] Kaplun S.: Low Reynolds number flow past a circular cylinder. J. of math. and mech. (1957), 595-603.

[8] Lamb, H.: On the uniform motion of a sphere through a viscous fluid. The London, Edinburgh, and Dublin Philosophical Magazine and Journal of Science 21(121) (1911) 112-121.

[9] Lamb H.: Hydrodynamics. Cambridge University Press (1932).

[10] Lee, S.H. and Leal, L.G.: Low-Reynolds-number flow past cylindrical bodies of arbitrary cross-sectional shape. J. fluid mech. 164 (1986), 401-427.

[11] Oseen, C.W.: Uber die Stokes sche Formel und Uber eine verwandte Aufgabe in der Hydrodynamik Arkiv Mat. Astron. och Fysik, 6(1) (1910).

[12] Oseen, C.W.: Neuere methoden und ergebnisse in der hydrodynamik. Leipzig: Akademische Verlagsgesellschaft mb H. (1927).

[13] Proudman, I. and Pearson, J.R.A.: Expansions at small Reynolds numbers for the flow past a sphere and a circular cylinder. J. fluid mech. 2(3) (1957), 237-262.

[14] Smith, D.J. and Gaffney, E.A. and Blake, J.R. and Kirkman-Brown, J.C.: Human sperm accumulation near surfaces: a simulation study. J. fluid mech. 621 (2009), 289-320.

[15] Stokes, G.G.: On the effect of the internal friction of fluids on the motion of pendulums. Pitt Press Cambridge (1851).

[16] Tomotika, S. and Aoi, T.: An expansion formula for the drag on a circular cylinder moving through a viscous fluid at small Reynolds numbers. The Quarterly Journal of Mechanics and Applied Mathematics 4(4) (1951), 401406.

[17] Tritton, D.J.: Experiments on the flow past a circular cylinder at low Reynolds numbers J. fluid mech. 6(4) (1959), 547-567. 
331 [18] Van Dyke, M.: An album of fluid motion Parabolic Press (1982).

332 [19] Yano, H. and Kieda, A.: An approximate method for solving two-dimensional 333 low-Reynolds number flow past arbitrary cylindrical bodies J. fluid mech. $334 \quad \mathbf{9 7}(\mathbf{1})(1980), 157-179$. 